\title{
Six-Minute Walking Distance Correlated with Memory and Brain Volume in Older Adults with Mild Cognitive Impairment: A Voxel-Based Morphometry Study
}

\author{
Hyuma Makizako a, d Hiroyuki Shimada ${ }^{a}$ Takehiko Doi ${ }^{a}$ d Hyuntae Park ${ }^{b}$ \\ Daisuke Yoshida ${ }^{a}$ Takao Suzukic
}

Sections for ${ }^{a}$ Health Promotion and ${ }^{b}$ Physical Functioning Activation, Center for Gerontology and Social Science, and 'Research Institute, National Center for Geriatrics and Gerontology, Obu, and dJapan Society for the Promotion of Science, Tokyo, Japan

Key Words

Exercise capacity · Logical memory · Visual memory · Brain atrophy · Fitness · Walking ·

Cognitive impairment

\begin{abstract}
Background/Aims: High fitness levels play an important role in maintaining memory function and delaying the progression of structural brain changes in older people at risk of developing dementia. However, it is unclear which specific regions of the brain volume are associated with exercise capacity. We investigated whether exercise capacity, determined by a 6-min walking distance (6MWD), is associated with measures of logical and visual memory and where gray matter regions correlate with exercise capacity in older adults with mild cognitive impairment (MCI). Methods: Ninety-one community-dwelling older adults with MCI completed a 6-min walking test, structural magnetic resonance imaging scanning, and memory tests. The Wechsler Memory Scale-Revised Logical Memory and Rey-Osterrieth Complex Figure Tests were used to assess logical and visual memory, respectively. Results: The logical and visual memory tests were positively correlated with the 6MWD $(p<0.01)$. Poor performance in the 6MWD was correlated with a reduced cerebral gray matter volume in the left middle temporal gyrus, middle occipital gyrus, and hippocampus in older adults with MCI. Conclusions: These results suggest that a better 6MWD performance may be related to better memory function and the maintenance of gray matter volume in older adults with MCI.
\end{abstract}




\section{Introduction}

Mild cognitive impairment (MCI) is a heterogeneous condition associated with the transitional phase between normal cognitive aging and dementia [1]. Progression rates to dementia and Alzheimer's disease (AD) for individuals with MCI have been reported as being in the range of 6-25\% per year [2]. MCI may be the optimum stage at which to intervene with preventive therapies.

Increased physical activity and higher aerobic fitness levels, defined as cardiorespiratory fitness, have been associated with the maintenance of cognitive function and a decreased risk for developing dementia [3,4]. Recent randomized controlled trials (RCTs) of aerobic exercise for healthy older adults provided evidence that participation in exercise programs involving aerobic exercise leads to an improvement in cognitive function [5] and a greater brain volume in specific regions, e.g. in the prefrontal cortex [6] and hippocampus [7]. Previous crosssectional studies have suggested that higher fitness levels associated with greater brain volumes in these regions were characteristic among healthy older adults [8, 9]. Some longitudinal studies have shown supportive results of the assumption that a greater physical activity predicts a stable cognitive function $[10,11]$ and gray matter volume [12].

Physical activity and exercise interventions can have a positive effect on cognitive function in older adults and even in those in the MCI stage $[13,14]$. In addition, a recently proposed RCT will examine the effects of a moderate physical activity program on delaying the progression of structural brain changes in older adults with MCI [15]. These studies suggest that a higher exercise capacity plays an important role in maintaining cognitive function and delaying structural brain changes in MCI. However, it is unclear which specific brain regions are associated with exercise capacity performance in older adults with MCI.

We investigated whether a 6-min walking distance (6MWD), to be established as exercise capacity performance, is associated with measures of gray matter volume in older adults with $\mathrm{MCI}$. The 6-min walking test (6MWT) is useful for predicting the maximal oxygen uptake related to cardiorespiratory fitness [16] and is easily administered in clinical settings [17]. The relationship between a 6MWD and memory performance was also examined in this study. A decline in memory performance represents a typical clinical sign of AD and can be observed 10 years prior to the expected symptom onset of AD [18]. In addition, poor memory performance and a lower gray matter volume in the medial temporal area, including the hippocampus, could predict progression to AD in older individuals with MCI [19, 20]. Maintaining exercise capacity may be related to a better memory performance and less brain atrophy in MCI subjects, and this positive relation may contribute to decreasing the risk of progression to AD. However, few studies have reported associations between fitness performance and memory performance in MCI subjects. We hypothesized that a better exercise capacity performance would correlate with a better memory performance and a greater brain volume among MCI subjects. A high exercise capacity may be sustained by a physically active lifestyle; this is potentially an important pathway for maintaining a healthy brain, both in terms of size and reduced damage.

\section{Participants and Methods}

\section{Participants}

Subjects in this study were recruited from our volunteer databases $(n=1,543)$, which included elderly individuals ( $\geq 65$ years old). Participants had to be community-dwelling adults aged $\geq 65$ years. Furthermore, all participants were required to meet the definition of MCI based on the Petersen criteria (not normal cognitive function for age, not demented, and 
Makizako et al.: Six-Minute Walking Distance Correlated with Memory and Brain Volume in Older Adults with Mild Cognitive Impairment: A Voxel-Based Morphometry Study

Table 1. Demographic and health characteristics $(\mathrm{n}=91)$

\begin{tabular}{lc} 
Age, years & $74.2 \pm 6.3$ \\
Female gender & $47(51.6)$ \\
BMI & $23.2 \pm 3.2$ \\
Diagnosis & $40(44.0)$ \\
$\quad$ Hypertension & $8(8.8)$ \\
$\quad$ Diabetes mellitus & $33(36.3)$ \\
$\quad$ Medication, $\geq 3$ & $3.6 \pm 3.1$ \\
Mental status & $27.0 \pm 1.9$ \\
$\quad$ GDS, points & $4.9 \pm 0.3$ \\
$\quad$ MMSE, points & $1.1 \pm 0.3$ \\
Physical status & \\
$\quad$ Instrumental self-maintenance ${ }^{\text {a }}$, points & \\
$\quad$ Walking speed, m/s & \\
\hline$\quad$ Values are mean \pm SD or number (percentage). GDS = Geriatric \\
Depression Scale. \\
$\quad$ The Tokyo Metropolitan Institute of Gerontology Index of \\
Competence subscale (0-5). \\
\hline
\end{tabular}

essentially normal functional activities) [21]. A total of 528 potential participants exhibiting a Clinical Dementia Rating score of 0.5 or a subjective memory complaint were enrolled in the first eligibility assessment. Of these, 135 participants underwent the second eligibility assessment, including neuropsychological tests, physical performance tests, face-to-face interviews, and magnetic resonance imaging (MRI) scans. The inclusion criteria required that the participants were $\geq 65$ years old, lived independently in the community (i.e., had no impairment of activities of daily living), were Japanese speaking with sufficient hearing and visual acuity to participate in the examinations, and had general cognitive function (MiniMental State Examination [22]) scores between 24 and 30. Exclusion criteria were a history of major psychiatric illness (e.g. schizophrenia or bipolar disorder), other serious neurological or musculoskeletal diagnoses, and clinical depression (Geriatric Depression Scale [23] score $\geq 10$ ). In addition, we excluded 9 participants who could not perform the physical performance tests and did not meet satisfactory requirements for the MRI scan. Finally, 91 participants complied with the inclusion criteria, and their data were analyzed in the present study. This study was approved by the Ethics Committee of the National Center for Geriatrics and Gerontology, and all participants provided written informed consent. Table 1 summarizes the characteristics of the participants.

\section{Logical and Visual Memory}

Logical and visual memory performances were in a standardized format and were administered by licensed, well-trained clinical speech therapists.

The Wechsler Memory Scale-Revised (WMS-R) Logical Memory (LM) [24] was used to assess logical memory. The WMS-R LM subtest requires the examiner to read aloud two short stories to the participant, each with 25 content units. In this study, stories from the Japanese version of the WMS-R LM test were used. After each story, the participant was asked to repeat the story immediately as close to verbatim as possible (immediate recall, Logical Memory-I). The recall was recorded verbatim and scored later according to the manual guidelines. After a 30-min delay, the examiner asked the subject to repeat each of the two stories once again for the delayed recall measure (delayed recall, Logical Memory-II).

The Rey-Osterrieth Complex Figure Test (ROCFT) [25] was used to assess visual memory. The ROCFT is a widely used instrument for assessing visual memory. The participants were 
Makizako et al.: Six-Minute Walking Distance Correlated with Memory and Brain Volume in Older Adults with Mild Cognitive Impairment: A Voxel-Based Morphometry Study

requested to copy the ROCFT figure and reproduce it immediately and again after a 30-min delay. They were not informed that they would be asked to recall the figure. The participants were allowed as much time as they needed for both copy and recall. During the retention interval, unrelated tests (e.g. Mini-Mental State Examination) were administered. The drawings were scored based on a 36-point scoring system.

\section{Six-Minute Walking Test}

We used the 6MWT to quantitatively measure participants' exercise capacity. The 6MWT measures the maximum distance that a person can walk in $6 \mathrm{~min}$. The 6MWT is a modification of the 12-min walk/run test originally developed by Cooper [26] and is commonly used as an assessment of exercise capacity. The 6MWT is useful for predicting the maximal oxygen uptake related to cardiorespiratory fitness and is easily administered in clinical settings [17]. The 6MWT was assessed by licensed, well-trained physical therapists. The participants were instructed to walk from one end of a 10-meter course to the other and back again as many times as possible in $6 \mathrm{~min}$, while under the supervision of a physical therapist. After each minute, participants were informed of the time elapsed and were given standardized encouragement. The distance (meters) walked in 6 min was recorded.

\section{MRI Procedure}

MRI was performed using a 1.5-tesla system (Magnetom Avanto; Siemens, Germany). Three-dimensional volumetric acquisition of a T1-weighted gradient-echo sequence was then used to produce a gapless series of thin sagittal sections using a magnetization preparation rapid-acquisition gradient-echo sequence (repetition time, 1,700 ms; echo time, 4.0 ms; flip angle, $15^{\circ}$; acquisition matrix, $256 \times 256$; slice thickness, $1.25 \mathrm{~mm}$ ). Tissue segmentation, regulation, registration, and normalization were conducted in the voxel-based morphometry (VBM) 8 toolbox (http://dbm.neuro.uni-jena.de/vbm/), which is incorporated in the SPM8 software (http://www.fil.ion.ucl.ac.uk/spm/), running on MATLAB R2010a (Mathworks). Diffeomorphic Anatomical Registration using Exponentiated Lie Algebra (DARTEL) [27] was conducted for the image analysis. The normalized images were transformed into the Montreal Neurological Institute (MNI) space. The gray matter images were then smoothened using a Gaussian kernel of $12 \mathrm{~mm}$ full width at half maximum.

\section{Statistical and VBM Analyses}

We calculated Pearson correlation coefficients, assessing simple relationships between memory tests and the 6MWD. We used linear regression analyses to assess independent relationships between the variables, while controlling for age and sex to minimize the confounding influence of age-related changes in exercise capacity and memory performance. Standardized beta values were calculated. These statistical analyses were performed using SPSS for Windows, version 19.0. Statistical significance was set at 0.05 for these analyses.

In the VBM analysis, data preprocessing and analysis was performed with the VBM8 toolbox, which is incorporated in the SPM8 software. VBM [28] was applied to determine regions where gray matter density showed a positive correlation with exercise capacity assessed by the 6MWT. We performed a multiple regression analysis on the smoothed gray matter images in SPM8. Age and sex were included in the model as covariates. The statistical threshold was set to $\mathrm{p}<0.05$, corrected for multiple comparisons across the reduced search volume using the family-wise error rate (FWE), with an extent threshold of 40 voxels. The detection of labeled regions from coordinates in the results was conducted using the SPM Anatomy Toolbox [29]. 


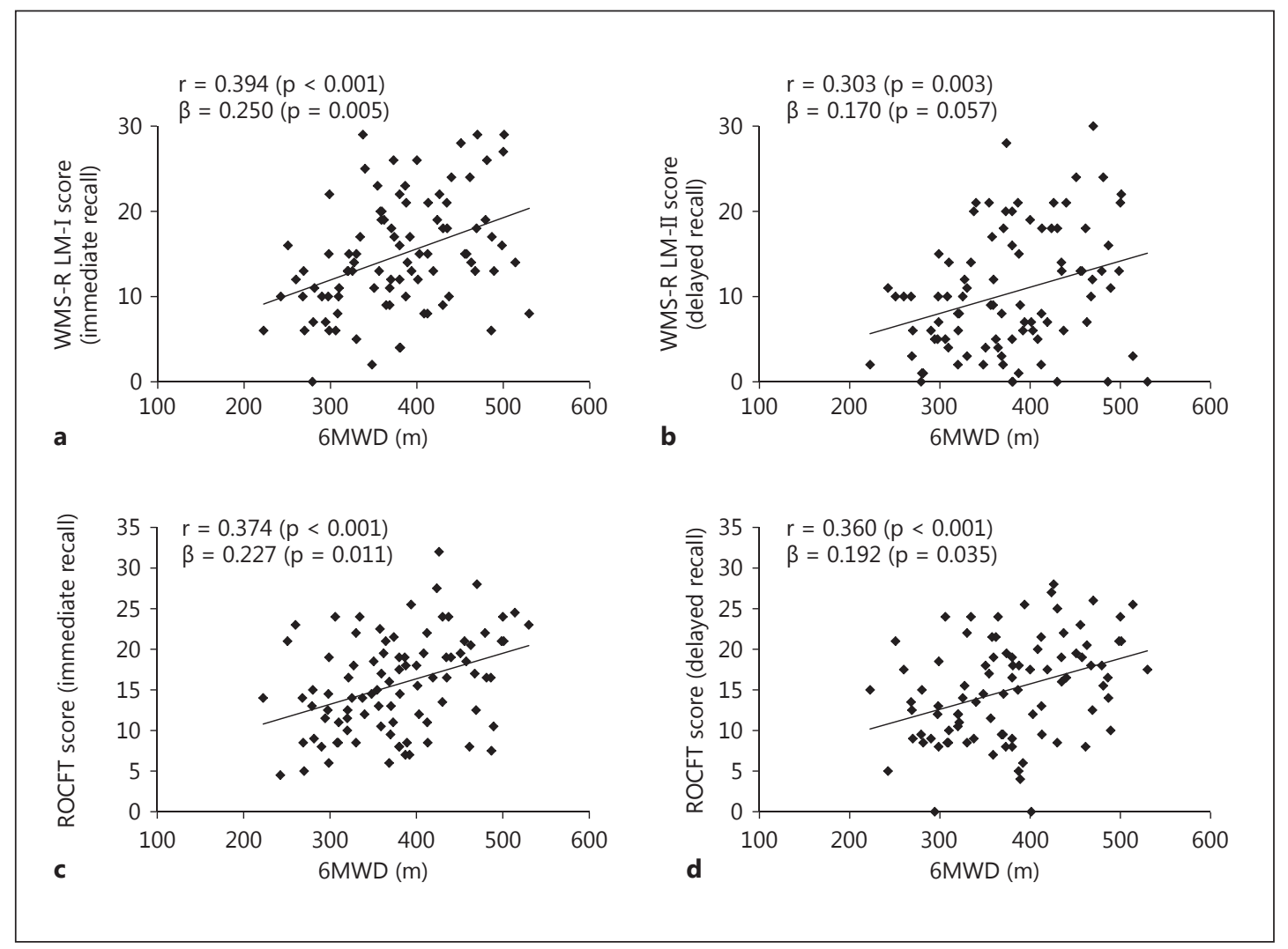

Fig. 1. Correlations between 6MWDs and memory performance tests. Pearson correlation coefficients (r) and standardized beta values (controlling for age and sex) are presented. a WMS-R LM-I (immediate recall). b WMS-R LM-II (delayed recall). c ROCFT (immediate recall). d ROCFT (delayed recall).

\section{Results}

Simple correlations were examined between the 6MWD and memory tests (fig. 1). Higher scores in all memory tests were significantly associated with a better performance on the 6MWT (WMS-R LM-I, $\mathrm{r}=0.394$, $\mathrm{p}<0.001$; WMS-R LM-II, $\mathrm{r}=0.303$, $\mathrm{p}=0.003$; ROCFT (immediate), $r=0.374, p<0.001$; ROCFT (delay), $r=0.360, p<0.001$ ). Although the relationship between the WMS-R LM-II and 6MWT was not statistically significant when the linear regression model was adjusted for age and sex (WMS-R LM-II, $\beta=0.170, p=0.057$ ), the other three memory tests were associated with the 6MWT even after controlling for age and sex [WMS-R LM-I, $\beta=0.250, \mathrm{p}=0.005$; ROCFT (immediate), $\beta=0.227$, $\mathrm{p}=0.011$; ROCFT (delay), $\beta=0.192, p=0.035]$.

Using multiple regression analysis in SPM8, we examined regions where gray matter density showed a positive correlation with exercise capacity. After adjusting for age and sex, gray matter density in the left middle temporal gyrus, middle occipital gyrus, and hippocampus showed positive correlations with the 6MWD (FWE, p < 0.05) (fig. 2). For the MNI coordinates, cluster size, peak $\mathrm{F}$ values, and $\mathrm{Z}$ values, please refer to table 2 . Figure 3 shows the highly linear relationship between 6MWD and adjusted gray matter density in the left hippocampus. 
Makizako et al.: Six-Minute Walking Distance Correlated with Memory and Brain Volume in Older Adults with Mild Cognitive Impairment: A Voxel-Based Morphometry Study

Left middle temporal gyrus $[x=-59, y=-6, z=-12]$

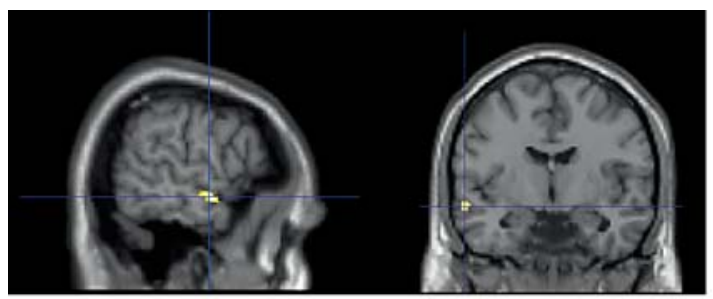

Left middle temporal gyrus $[x=-54, y=5, z=-21]$

Left middle occipital gyrus $[x=-44, y=-85, z=13]$
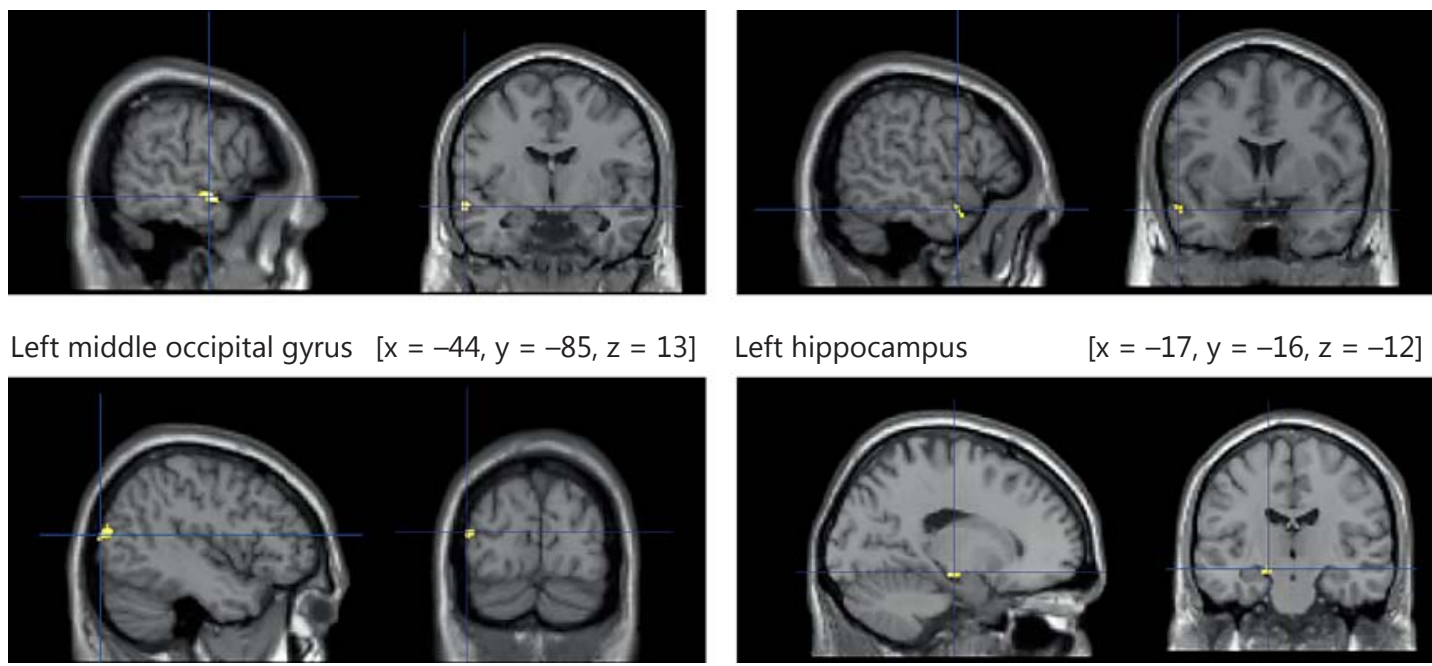

Left hippocampus

$[x=-17, y=-16, z=-12]$

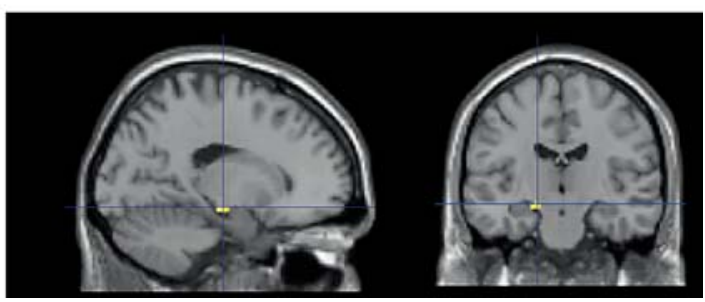

Fig. 2. Brain regions showing an association between a better performance in the 6MWT and a greater gray matter volume. After adjusting for age and sex, gray matter density in the left middle temporal gyrus, middle occipital gyrus, and hippocampus showed positive correlations with the 6MWD.

Fig. 3. Correlation between VBM response in the left hippocampus peak voxel (adjusted for effects of age and sex) and the 6MWD.

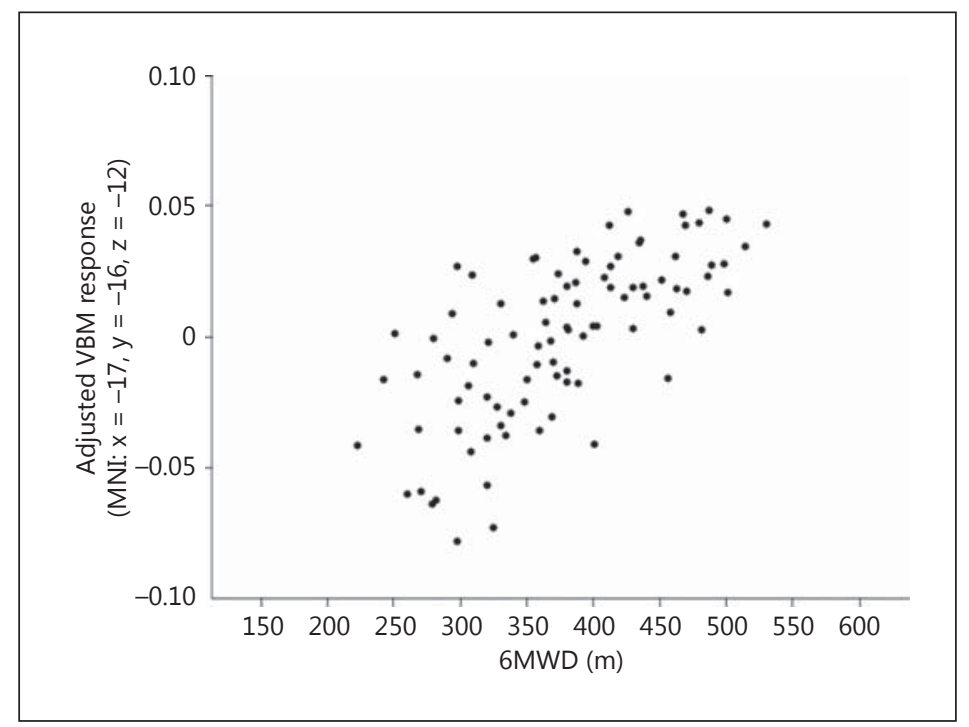

\section{Discussion}

We confirmed that memory performance was significantly positively associated with exercise capacity as assessed by a 6MWD in older adults with MCI. After adjusting for age and sex, gray matter density in the left middle temporal gyrus, middle occipital gyrus, and hippocampus showed positive correlations with exercise capacity.

Previous epidemiological studies in aging populations have suggested beneficial effects of increased physical activity on brain health and function [30, 31]. In a cross-sectional study of 75 healthy older individuals, a positive association between physical activity and memory performance was reported [32]. An interventional study among older adults indicated a 
Table 2. VBM results of a 6MWD and volume regions of interest after adjusting for age and sex

\begin{tabular}{|c|c|c|c|c|c|c|c|}
\hline \multirow[t]{2}{*}{ Location } & \multirow{2}{*}{$\begin{array}{l}\text { Cluster } \\
\text { size, K }\end{array}$} & \multirow[t]{2}{*}{ Peak F } & \multirow[t]{2}{*}{ Z-score } & \multirow[t]{2}{*}{ FWE, $p$} & \multicolumn{3}{|c|}{ MNI coordinates, mm } \\
\hline & & & & & $\mathrm{x}$-axis & $y$-axis & z-axis \\
\hline \multirow[t]{2}{*}{ Left middle temporal gyrus } & 79 & 32.81 & 5.13 & 0.004 & -59 & -6 & -12 \\
\hline & 27 & 27.58 & 4.74 & 0.024 & -54 & 5 & -21 \\
\hline Left middle occipital gyrus & 105 & 28.87 & 4.84 & 0.016 & -44 & -85 & 13 \\
\hline Left hippocampus & 46 & 29.54 & 4.89 & 0.013 & -17 & -16 & -12 \\
\hline
\end{tabular}

correlation between an increase of total physical activity and improved episodic memory after low- and medium-intensity physical training [33]. Pereira et al. [34] demonstrated that verbal memory performance was improved after completion of a 3-month aerobic exercise regime among adults aged 21-45 years. This improvement in verbal memory performance positively correlated with an improvement of the participants' cardiovascular fitness level and with the cerebral blood volume in the dentate gyrus of the hippocampus. These results support the present study, indicating associations between a greater 6MWD and a better memory function among older adults with MCI.

One advantage of the present results is the indication of the association between exercise capacity performance and gray matter volumes using MRI data among MCI subjects. In a large cross-sectional study of elderly subjects without dementia, physical fitness was highly and significantly associated with hippocampal volumes [8]. Another cross-sectional study also indicated that increased cardiorespiratory fitness was associated with a better preservation of gray matter volumes, particularly in the medial temporal lobes, including the hippocampus and parahippocampal gyrus [35]. Moreover, recent RCTs of aerobic exercise for older adults provided evidence for positive associations between aerobic exercise and greater brain volumes in specific regions. An RCT in a large cohort of older adults documented significantly larger hippocampal volumes after 1 year of aerobic exercise compared with the control intervention of simple stretching and toning [7]. The results of this study also confirmed that an increased exercise capacity performance was associated with greater brain volumes in specific regions, including the left middle temporal gyrus, middle occipital gyrus, and hippocampus even after adjusting for age and sex among MCI subjects.

A previous study using VBM analysis revealed that there was a significantly greater gray matter loss in converters from MCI to probable AD relative to nonconverters in the hippocampal area, inferior and middle temporal gyrus, posterior cingulate, and precuneus [36]. In a longitudinal study where individuals in late adulthood were followed up for 9 years, a greater physical activity predicted greater volumes of the frontal, occipital, entorhinal, and hippocampal regions [12]. Gray matter volumes in the medial temporal lobe, including the entorhinal, parahippocampal, and hippocampal regions, may contribute to the prediction of subsequent cognitive decline and conversion from MCI to AD [37], and may be important for maintaining memory function [38]. We demonstrated linear relationships between VBM response in the left hippocampus peak voxel and the 6MWD in figure 3. This association may indicate protective effects of exercise capacity on cognitive decline in older adults with MCI.

Recent interventional studies suggested that physical activity and aerobic exercise have beneficial effects on memory function. These effects are possibly mediated by gray matter volume and neurotrophic factors, especially brain-derived neurotrophic factor (BDNF) [7, 33], which is highly concentrated in the hippocampus [39] and is important for synaptic plasticity [40]. In a previous study including young adult males, both acute and chronic exercise improved medial temporal lobe function concomitant with increased concentrations of BDNF 
in the serum. This suggests a possible functional role for this neurotrophic factor in exerciseinduced cognitive enhancement [41]. Exercise has consistently been shown to enhance learning and persistently upregulate expression of BDNF in the hippocampus of rodent models $[42,43]$. These previous results may support the present findings that exercise capacity is related to brain volume including the medial temporal lobe. However, this study did not provide evidence of mechanisms for protective effects of aerobic fitness on brain volume through neurotrophic factors. Future studies are needed to provide insight into how mechanisms that increase fitness may enhance cognition, especially memory, and prevent age-related structural brain changes.

Several possible limitations should be considered when interpreting our findings. We are conscious of the limitations of our cross-sectional design. Longitudinal and interventional studies should be designed to clarify the relationship between exercise capacity and cognitive function among MCI subjects. In addition, we recognize that there is important information regarding the effect of exercise capacity on the conversion rate from MCI to AD. Our results indicate that a higher exercise capacity may be related to a better memory function and a greater gray matter volume in several brain regions. This has been found in other studies including healthy older adults [44] or AD patients [35]. However, in the present and previous studies, different methods of assessment were used to identify fitness levels. Previous studies that examined the relationships between aerobic fitness and brain volume used the measurement of peak oxygen consumption [35, 44]. We assessed participants' exercise capacity with the 6MWT. This measure is widely used in clinical settings to identify exercise capacity and is associated with peak oxygen consumption in older adults. We did not include data from healthy older persons and patients with AD in the present study. Additional neurological analyses that include data from healthy older adults and AD patients are needed to determine the relationships between exercise capacity and brain changes in AD-related processes. Although a previous neuroimaging study suggested that the apolipoprotein E $\varepsilon 4$ genotype in MCI might be associated with structural changes typically found in the early stages of AD [45], our data did not consider the effects of genetic factors, such as the presence of the apolipoprotein E risk allele.

In conclusion, a higher exercise capacity measured by the 6MWT is associated with a better memory function and a greater gray matter density, including the left middle temporal gyrus, middle occipital gyrus, and hippocampus in older adults with MCI. To strengthen our findings, future studies are required to examine the effects of intervention on exercise capacity and the related change in brain volume in the specific regions and memory function among MCI subjects.

\section{Acknowledgments}

We would like to thank the Obu City Office for their assistance with participant recruitment. We would also like to thank the technical staff from the Department of Radiology, National Hospital for Geriatric Medicine, National Center for Geriatrics and Gerontology for MRI data acquisition. This work was supported by a grant from the Japanese Ministry of Health, Labour and Welfare (Project for optimizing long-term care; B-3) to T.S. and a grantin-aid for JSPS Fellows (23-9862) from the Japan Society for the Promotion of Science to H.M.

\section{Disclosure Statement}

There are no conflicts of interest. 
Makizako et al.: Six-Minute Walking Distance Correlated with Memory and Brain Volume in Older Adults with Mild Cognitive Impairment: A Voxel-Based Morphometry Study

\section{References}

1 Petersen RC, Doody R, Kurz A, Mohs RC, Morris JC, Rabins PV, Ritchie K, Rossor M, Thal L, Winblad B: Current concepts in mild cognitive impairment. Arch Neurol 2001;58:1985-1992.

-2 Petersen RC, Stevens JC, Ganguli M, Tangalos EG, Cummings JL, DeKosky ST: Practice parameter: early detection of dementia: mild cognitive impairment (an evidence-based review). Report of the Quality Standards Subcommittee of the American Academy of Neurology. Neurology 2001;56:1133-1142.

-3 Barnes DE, Yaffe K, Satariano WA, Tager IB: A longitudinal study of cardiorespiratory fitness and cognitive function in healthy older adults. J Am Geriatr Soc 2003;51:459-465.

-4 Larson EB, Wang L, Bowen JD, McCormick WC, Teri L, Crane P, Kukull W: Exercise is associated with reduced risk for incident dementia among persons 65 years of age and older. Ann Intern Med 2006;144:73-81.

-5 Voss MW, Nagamatsu LS, Liu-Ambrose T, Kramer AF: Exercise, brain, and cognition across the life span. J Appl Physiol 2011;111:1505-1513.

-6 Colcombe SJ, Erickson KI, Scalf PE, Kim JS, Prakash R, McAuley E, Elavsky S, Marquez DX, Hu L, Kramer AF: Aerobic exercise training increases brain volume in aging humans. J Gerontol A Biol Sci Med Sci 2006;61: 1166-1170.

-7 Erickson KI, Voss MW, Prakash RS, Basak C, Szabo A, Chaddock L, Kim JS, Heo S, Alves H, White SM, Wojcicki TR, Mailey E, Vieira VJ, Martin SA, Pence BD, Woods JA, McAuley E, Kramer AF: Exercise training increases size of hippocampus and improves memory. Proc Natl Acad Sci USA 2011;108:3017-3022.

-8 Erickson KI, Prakash RS, Voss MW, Chaddock L, Hu L, Morris KS, White SM, Wojcicki TR, McAuley E, Kramer AF: Aerobic fitness is associated with hippocampal volume in elderly humans. Hippocampus 2009;19:10301039.

9 Weinstein AM, Voss MW, Prakash RS, Chaddock L, Szabo A, White SM, Wojcicki TR, Mailey E, McAuley E, Kramer AF, Erickson KI: The association between aerobic fitness and executive function is mediated by prefrontal cortex volume. Brain Behav Immun 2012;26:811-819.

10 Yaffe K, Fiocco AJ, Lindquist K, Vittinghoff E, Simonsick EM, Newman AB, Satterfield S, Rosano C, Rubin SM, Ayonayon HN, Harris TB: Predictors of maintaining cognitive function in older adults: the Health ABC study. Neurology 2009;72:2029-2035.

-11 Chang M, Jonsson PV, Snaedal J, Bjornsson S, Saczynski JS, Aspelund T, Eiriksdottir G, Jonsdottir MK, Lopez OL, Harris TB, Gudnason V, Launer LJ: The effect of midlife physical activity on cognitive function among older adults: AGES-REYKJAVIK Study. J Gerontol A Biol Sci Med Sci 2010;65:1369-1374.

$\checkmark 12$ Erickson KI, Raji CA, Lopez OL, Becker JT, Rosano C, Newman AB, Gach HM, Thompson PM, Ho AJ, Kuller LH: Physical activity predicts gray matter volume in late adulthood: the Cardiovascular Health Study. Neurology 2010;75:1415-1422.

13 Baker LD, Frank LL, Foster-Schubert K, Green PS, Wilkinson CW, McTiernan A, Plymate SR, Fishel MA, Watson GS, Cholerton BA, Duncan GE, Mehta PD, Craft S: Effects of aerobic exercise on mild cognitive impairment: a controlled trial. Arch Neurol 2010;67:71-79.

14 van Uffelen JG, Chinapaw MJ, van Mechelen W, Hopman-Rock M: Walking or vitamin B for cognition in older adults with mild cognitive impairment? A randomised controlled trial. Br J Sports Med 2008;42:344-351.

-15 Cyarto EV, Lautenschlager NT, Desmond PM, Ames D, Szoeke C, Salvado O, Sharman MJ, Ellis KA, Phal PM, Masters CM, Rowe CC, Martins RN, Cox KL: Protocol for a randomized controlled trial evaluating the effect of physical activity on delaying the progression of white matter changes on MRI in older adults with memory complaints and mild cognitive impairment: the AIBL Active trial. BMC Psychiatry 2012;12:167.

-16 Cataneo DC, Kobayasi S, Carvalho LR, Paccanaro RC, Cataneo AJ: Accuracy of six minute walk test, stair test and spirometry using maximal oxygen uptake as gold standard. Acta Cir Bras 2010;25:194-200.

-17 Steffen TM, Hacker TA, Mollinger L: Age- and gender-related test performance in community-dwelling elderly people: Six-Minute Walk Test, Berg Balance Scale, Timed Up \& Go Test, and gait speeds. Phys Ther 2002;82: 128-137.

18 Bateman RJ, Xiong C, Benzinger TL, Fagan AM, Goate A, Fox NC, Marcus DS, Cairns NJ, Xie X, Blazey TM, Holtzman DM, Santacruz A, Buckles V, Oliver A, Moulder K, Aisen PS, Ghetti B, Klunk WE, McDade E, Martins RN, Masters CL, Mayeux R, Ringman JM, Rossor MN, Schofield PR, Sperling RA, Salloway S, Morris JC: Clinical and biomarker changes in dominantly inherited Alzheimer's disease. N Engl J Med 2012;367:795804.

19 Prins ND, van der Flier WM, Brashear HR, Knol DL, van de Pol LA, Barkhof F, Scheltens P: Predictors of progression from mild cognitive impairment to dementia in the placebo-arm of a clinical trial population. J Alzheimers Dis 2013;36:79-85.

20 Korf ES, Wahlund LO, Visser PJ, Scheltens P: Medial temporal lobe atrophy on MRI predicts dementia in patients with mild cognitive impairment. Neurology 2004;63:94-100.

21 Petersen RC: Mild cognitive impairment as a diagnostic entity. J Intern Med 2004;256:183-194.

-22 Folstein MF, Folstein SE, McHugh PR: 'Mini-mental state'. A practical method for grading the cognitive state of patients for the clinician. J Psychiatr Res 1975;12:189-198.

23 Yesavage JA: Geriatric depression scale. Psychopharmacol Bull 1988;24:709-711.

24 Wechsler D: Wechsler Memory Scale-Revised Manual. San Antonio, The Psychological Corporation, 1987.

25 Meyers J, Meyers K: Rey Complex Figure Test and Recognition Trial: Professional Manual. Odessa, Psychological Assessment Resources, 1995. 
Makizako et al.: Six-Minute Walking Distance Correlated with Memory and Brain Volume

-26 Cooper KH: A means of assessing maximal oxygen intake. Correlation between field and treadmill testing JAMA 1968;203:201-204.

27 Ashburner J: A fast diffeomorphic image registration algorithm. Neuroimage 2007;38:95-113.

28 Ashburner J, Friston KJ: Unified segmentation. Neuroimage 2005;26:839-851.

29 Eickhoff SB, Heim S, Zilles K, Amunts K: Testing anatomically specified hypotheses in functional imaging using cytoarchitectonic maps. Neuroimage 2006;32:570-582.

30 Ravaglia G, Forti P, Lucicesare A, Pisacane N, Rietti E, Bianchin M, Dalmonte E: Physical activity and dementia risk in the elderly: findings from a prospective Italian study. Neurology 2008;70:1786-1794.

-31 Sattler C, Erickson KI, Toro P, Schroder J: Physical fitness as a protective factor for cognitive impairment in a prospective population-based study in Germany. J Alzheimers Dis 2011;26:709-718.

-32 Floel A, Ruscheweyh R, Kruger K, Willemer C, Winter B, Volker K, Lohmann H, Zitzmann M, Mooren F, Breitenstein C, Knecht S: Physical activity and memory functions: are neurotrophins and cerebral gray matter volume the missing link? Neuroimage 2010;49:2756-2763.

-33 Ruscheweyh R, Willemer C, Kruger K, Duning T, Warnecke T, Sommer J, Volker K, Ho HV, Mooren F, Knecht S, Floel A: Physical activity and memory functions: an interventional study. Neurobiol Aging 2011;32:13041319.

34 Pereira AC, Huddleston DE, Brickman AM, Sosunov AA, Hen R, McKhann GM, Sloan R, Gage FH, Brown TR, Small SA: An in vivo correlate of exercise-induced neurogenesis in the adult dentate gyrus. Proc Natl Acad Sci USA 2007;104:5638-5643.

-35 Honea RA, Thomas GP, Harsha A, Anderson HS, Donnelly JE, Brooks WM, Burns JM: Cardiorespiratory fitness and preserved medial temporal lobe volume in Alzheimer disease. Alzheimer Dis Assoc Disord 2009;23:188197.

-36 Chetelat G, Landeau B, Eustache F, Mezenge F, Viader F, de la Sayette V, Desgranges B, Baron JC: Using voxelbased morphometry to map the structural changes associated with rapid conversion in MCI: a longitudinal MRI study. Neuroimage 2005;27:934-946.

-37 Ferreira LK, Diniz BS, Forlenza OV, Busatto GF, Zanetti MV: Neurostructural predictors of Alzheimer's disease: a meta-analysis of VBM studies. Neurobiol Aging 2011;32:1733-1741.

-38 Apostolova LG, Morra JH, Green AE, Hwang KS, Avedissian C, Woo E, Cummings JL, Toga AW, Jack CR Jr, Weiner MW, Thompson PM: Automated 3D mapping of baseline and 12-month associations between three verbal memory measures and hippocampal atrophy in 490 ADNI subjects. Neuroimage 2010;51:488-499.

-39 Phillips HS, Hains JM, Laramee GR, Rosenthal A, Winslow JW: Widespread expression of BDNF but not NT3 by target areas of basal forebrain cholinergic neurons. Science 1990;250:290-294.

40 Kang H, Schuman EM: Long-lasting neurotrophin-induced enhancement of synaptic transmission in the adult hippocampus. Science 1995;267:1658-1662.

-41 Griffin EW, Mullally S, Foley C, Warmington SA, O'Mara SM, Kelly AM: Aerobic exercise improves hippocampal function and increases BDNF in the serum of young adult males. Physiol Behav 2011;104:934-941.

-42 Gobbo OL, O'Mara SM: Exercise, but not environmental enrichment, improves learning after kainic acidinduced hippocampal neurodegeneration in association with an increase in brain-derived neurotrophic factor. Behav Brain Res 2005;159:21-26.

43 Griffin EW, Bechara RG, Birch AM, Kelly AM: Exercise enhances hippocampal-dependent learning in the rat: evidence for a BDNF-related mechanism. Hippocampus 2009;19:973-980.

-44 Colcombe SJ, Erickson KI, Raz N, Webb AG, Cohen NJ, McAuley E, Kramer AF: Aerobic fitness reduces brain tissue loss in aging humans. J Gerontol A Biol Sci Med Sci 2003;58:176-180.

45 Thomann PA, Roth AS, Dos Santos V, Toro P, Essig M, Schroder J: Apolipoprotein E polymorphism and brain morphology in mild cognitive impairment. Dement Geriatr Cogn Disord 2008;26:300-305. 